\section{Evaluation of Nasal Mucociliary Clearance Time in COVID-19 Patients}

\author{
Ayse Çeçen, MD, Cem Bayraktar, MD, \\ Abdulkadir Özgür, MD, Gökhan Akgül, MD, \\ and Ögür Günal, $M D$
}

Background: Coronavirus Disease 2019 (COVID-19) is a viral pandemic emerging in East Asia and spreading rapidly to the rest of the world and continuing in our country. The number of studies examining the symptoms of the otolaryngology system and organs in COVID-19 patients is limited. The purpose of this study is to show whether COVID-19 infections cause any changes in nasal physiology in adult patients by measuring the mucociliary clearance (MCC) time with the saccharin test.

Methods: One hundred one patients with laboratory-confirmed COVID-19 infection (group 1, $\mathrm{n}=42$ smokers and group 2, $\mathrm{n}=59$ nonsmokers) and 87 individuals without COVID-19 infection as the control group (group 3, $\mathrm{n}=33$ smokers and group 4, $\mathrm{n}=54$ nonsmokers) were included in the study. A saccharin test was used to evaluate the nasal MCC time.

Results: Mucociliary clearance test averages of the groups were determined as $473.571 \pm 263.684,442.966 \pm 228.463,468.333 \pm$ $267.367,412.629 \pm 192.179$ seconds, respectively. When the test durations were examined, the group with the most prolonged $\mathrm{MCC}$ duration was determined as the smoking COVID $(+)$ patient group (473.571 \pm 263.684 seconds). The second group, with the most prolonged MCC duration, was determined as the smoking control group ( $468.333 \pm 267.367$ seconds). No statistically significant difference was found in intergroup mucociliary clearance time $(P=0.760)$.

Conclusion: Although the authors found that smoking and viral infections prolong the MCC duration in our study, they did not find a statistically significant difference between the groups in terms of MCC duration.

Key Words: Coronavirus disease 2019, mucociliary clearance time, smokers, viral infection

From the Department of Otolaryngology, Samsun Education and Research Hospital, Samsun, Turkey.

Received October 27, 2020.

Accepted for publication February 20, 2021.

Address correspondence and reprint requests to Ayse Çeçen, MD, Department of Otorhinolaryngology, University of Health Sciences, Samsun Health Practices and Research Center, Kadiköy Mah. Park Sok. No. 199, PB: 55090, Ilkadim, Samsun, Turkey;

E-mail: aysebel55@hotmail.com

Before starting the study, we take permission from ethical board of Samsun Training and Research Hospital.

The authors have informed consent for the study.

The authors report no conflicts of interest

Supplemental digital contents are available for this article. Direct URL citations appear in the printed text and are provided in the HTML and PDF versions of this article on the journal's Web site (www.jcraniofacialsurgery.com).

Copyright ( 12021 by Mutaz B. Habal, MD

ISSN: $1049-2275$

DOI: $10.1097 /$ SCS.0000000000007699
A $t$ the end of 2019, a novel member of the human RNA coronavirus identified as Severe Acute Respiratory Syndrome Coronavirus 2 (SARS-CoV-2) was identified in Wuhan, China. This novel coronavirus has been recognized as a cause of lower respiratory tract infections. The International Virus Taxonomy Committee has formally named it a severe acute respiratory syndrome-coronavirus 2 (SARS-CoV-2). On February 11, 2020, the World Health Organization (WHO) named the disease caused by SARS-CoV-2 as coronavirus disease 2019 (COVID-19). The virus, which has a high potential of human-to-human transmission, caused a rapid COVID-19 epidemic in China and then caused a global pandemic. $^{1,2}$

An infected person may experience symptoms after an incubation period that can range from about 2 to 14 days (there have been rare cases of an incubation period of 29 days), during which time the person can still be contagious. Precautions such as careful personal hygiene, frequent hand washing, wearing masks, and social distancing should be taken to limit transmission. ${ }^{3}$ In most cases, spread between humans occurs through respiratory droplets that are spread through an infected person's coughing or sneezing and then inhaled by a nearby healthy person. ${ }^{4,5}$ You can become infected if you touch your mouth, nose, or eyes after touching surfaces or objects where the virus has been found. ${ }^{5}$

In the COVID-19 pandemic, physicians and dentists, who are particularly at risk due to their relationship with anatomical regions where viral load is high, are among the most exposed people to the virüs. In order to reduce the risk of infection, preventive triage method should be used. The triage prevention method allows clinicians to identify patients potentially infected with the coronavirus. Telemedicine, smartphones, and applications minimize the risk of cross-contamination by minimizing unnecessary patientdoctor contact, while identifying emergency and priority situations. $^{5}$

Cleaning foreign particles of the nasal mucosa and keeping the mucosa moist is necessary for normal nasal physiology. These abilities of the respiratory mucosa depend on ciliary activity and regeneration of airway secretions. Together these are known as the mucociliary activity. Mucociliary clearance (MCC) is the most important defense mechanism of the nasal epithelium. The harmful foreign material is involved by this mucus layer and removed from the nasal cavity with ciliary movements. ${ }^{6,7}$

Coloring agents such as saccharin, radionuclide substances, and methylene blue were used for MCC measurement. The saccharine clearance test, which is an in vivo technique for the evaluation of nasal MCC, was defined by Andersen et $\mathrm{al}^{8}$ in 1974 and modified by Rutland and Cole. ${ }^{9}$ The saccharine clearance test is preferred more often than the other methods used for this measurement because it is cheap, easy, and reliable. The average test time varies between 7 and 15 minutes and transport time longer than 30 minutes indicates that nasal MCC is impaired. ${ }^{10}$

Mucociliary clearance may be affected by ambient temperature, humidity, partial $\mathrm{O} 2$ pressure, $\mathrm{pH}$, trauma, sulfur dioxide, formaldehyde, ozone, chlorine, smoking, viral infections, chronic sinusitis, chronic and allergic rhinitis, adenoid hypertrophy, cystic fibrosis, chronic bronchitis, septum deviation, surgery, bronchial asthma, and diabetes. ${ }^{6,7}$

Nasal MCC duration is prolonged in viral respiratory tract infections and chronic sinusitis. ${ }^{11}$ Since COVID-19 is also a viral infection, mucociliary clearance duration may be prolonged, and nasal symptoms may increase.

It is known that smoking causes changes in mucociliary clearance by decreasing the healthy cilia population and disrupting the viscoelasticity of mucus. ${ }^{6,7}$

The purpose of this study is to investigate whether the COVID19 infection, which was confirmed in the laboratory, causes the 
MCC to be affected, and to compare and reveal whether there is a relationship between smoking, disease duration, and lung involvement severity and clearance times with the control group.

\section{MATERIALS AND METHODS}

\section{Study Design}

The study was conducted between April 2020 and July 2020, in Samsun Training and Research Hospital, Department of Ear Nose and Throat Diseases and Department of Infectious Diseases Clinical Microbiology after obtaining the approval of the Samsun Training and Research Hospital Human Ethics Committee (Decision number: 2020/5/11). The study was carried under the rules of the Helsinki Declaration. Patients who were hospitalized during this study and agreed to participate in the study were included in the study. The study was conducted on patients hospitalized in the Department of Infectious Diseases and Clinical Microbiology. One hundred one patients with laboratory-confirmed COVID-19 infection (group 1, $\mathrm{n}=42$ smokers and group $2, \mathrm{n}=59$ nonsmokers) and 87 individuals without COVID-19 infection as the control group (group 3, $\mathrm{n}=33$ smokers and group $4, \mathrm{n}=54$ non-smokers) were included in the study. A written consent form was obtained from all patients.

\section{Patients}

One hundred eighty-eight subjects, including 101 patients with COVID-19 (COVID Group) and 87 healthy controls (Control Group), were included in this prospective study.

COVID-19 infection diagnosis is based on serological tests with COVID-19-specific IgM or IgG and/or reverse transcriptase-polymerase chain reaction (RT-PCR).

Researchers applied current tests to patients with appropriate protective equipment to avoid viral transmission. Age, gender, accompanying systemic disease, smoking status, and nasal surgery history of all patients were questioned. Those with nasal polyps, allergic rhinitis, chronic sinusitis, vasomotor rhinitis, sinonasal malignancy, major septal deviation, conchal hypertrophy, trauma, or epistaxis history in the last 48 hours, those who used drugs that may affect mucociliary function such as systemic and local decongestants, local nasal steroids, bronchodilators, anticholinergic agents in the last three months and patients who have had previous nasal surgery were not included in the study. Anterior rhinoscopic examinations of all patients were performed, and patients with nasal pathologies such as sinusitis, septum deviation, nasal polyposis that could cause nasal obstruction were excluded from the study. A control group was formed from healthy individuals of appropriate age and gender for the patient group. All patients included in the study underwent MCC tests with saccharine.

In this study, the saccharin test was performed by a single physician to prevent inter-observer variation in the measurement technique. One hour before the saccharin test, patients were asked to clean their nose by blowing their nose and not eating anything. Patients were instructed not to sniff, clean their nose, or sneeze during the test. The patients were placed in a sitting position with their head on the shoulders while breathing normally and were told not to lean forward. In the saccharin test, a 1/4 saccharin tablet was placed on the anterior end of the inferior concha with an alligator forceps, and patients were asked to inform when they had a taste. The time to the first sweet taste experience in the posterior nasopharynx was recorded as nasal MCC duration in seconds. We asked patients to swallow every 30 seconds and report when they tasted the saccharin. The information on how they would taste was not shared with the patients beforehand to increase test compliance and accuracy, and the patient was asked to experience and express it themself.
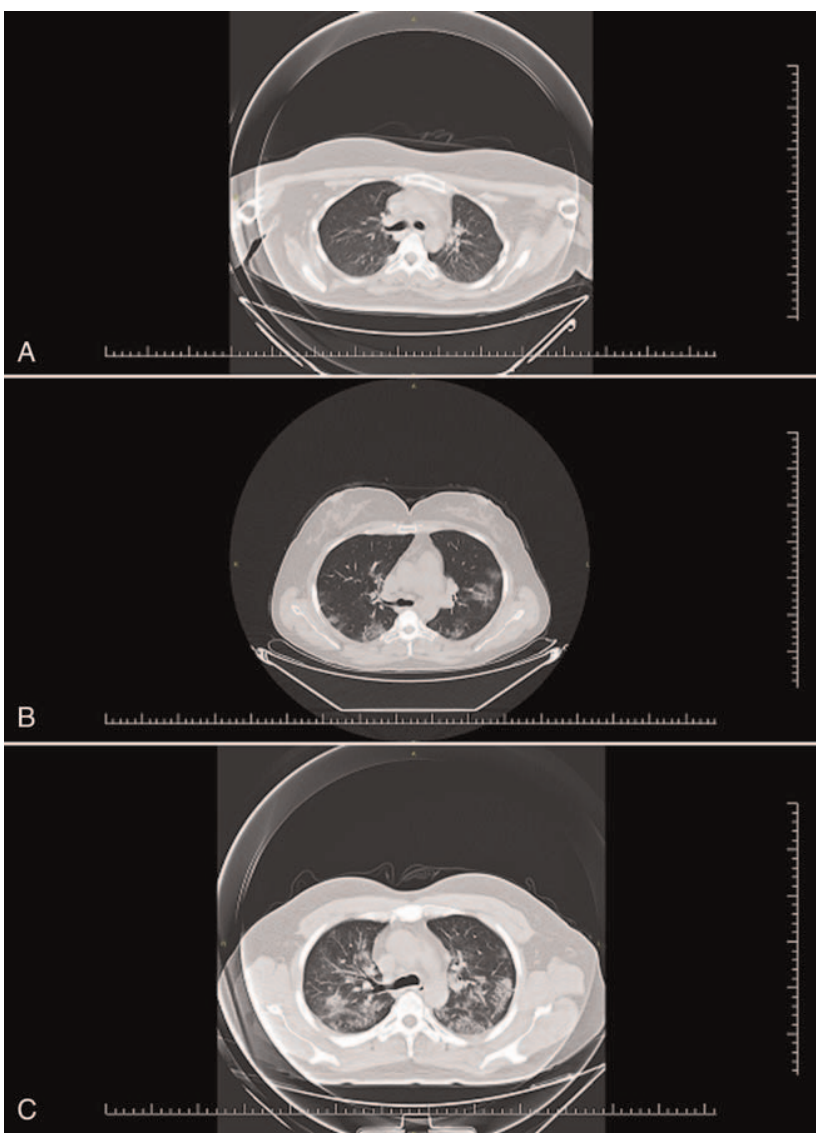

FIGURE 1. Routine Thoracic CT of COVID-19 patients. (A) Normal, (B) Focal, (C) Diffuse. COVID-19, coronavirus disease 2019.

Routine thoracic computed tomography (CT) of COVID-19 patients were grouped according to their normal, focal, and diffuse involvement, and the relationship between these groups and MCC was evaluated. Patients without pulmonary involvement were accepted as normal (Fig. 1A), those limited to one lung lobe as focal (Fig. 1B), and widespread involvement in both lungs was accepted as diffuse (Fig. 1C).

The patients were divided into 3 groups (group: 1, 1-3 days, group 2: 4-7 days, group 3: $8-14$ days) according to the day of the MCC test, and the relationship between these groups and MCC was evaluated.

\section{Statistical Methods}

Statistical Package for Social Science (SPSS) 22.0 software program (SPSS Inc., Chicago, IL) was used for statistical analysis. In data assessment, Kolmogr of Siminorv was used to test whether data normally distributed or not. Kruskal-Wallis test was used as a nonparametric test to compare the groups. Chi-square test was used to assess the intergroup difference between groups in gender. Pearson correlation test was used to compare mucociliary clearance and other variables. The significance level was taken as 0.05 .

\section{RESULTS}

The mean age of COVID groups (group 1:41.738 \pm 15.117 , group $2: 44.355 \pm 18.354$ ) and control groups (group 3:41.303 \pm 16.499 , group 4:46.037 \pm 15.819$)$ was years, respectively. A total of 188 subjects, including 101 patients (group 1: 16 females (\%38.1) and 26 males (\%61.9), group 2: 29 females (\%49.2), 30 males (\%50.8) 
having COVID-19 (COVID Group) and 87 (group 3: 9 females (\%27.3) and 24 males (\%72.7), group 4: 25 females (\%46.3),29 males (\%53.7)) healthy controls (Control Group), were included in this prospective study. The groups were similar in terms of age and gender $(P=0.376, P=0.183)$ (Supplementary Digital Content, Table 1, http://links.lww.com/SCS/C682).

Mucociliary clearance test averages of the groups were determined as $473.571 \pm 263.684, \quad 442.966 \pm 228.463, \quad 468.333 \pm$ $267.367,412.629 \pm 192.179$ seconds, respectively. When the test durations were examined, the group with the most prolonged MCC duration was determined as the smoking COVID $(+)$ patient group $(473.571 \pm 263.684$ seconds). The second group, with the most prolonged MCC duration, was determined as the smoking control group ( $468.333 \pm 267.367$ seconds). Although we found that smoking and viral infections prolong the MCC duration in our study, we did not find a statistically significant difference between the groups in terms of MCC duration $(P=0.760)$ (Supplementary Digital Content, Table 1, http://links.lww.com/SCS/C682).

Coronavirus Disease 2019 patients were grouped according to the degree of thoracic CT involvement, and the relationship between these groups and MCC was evaluated, and the result was not statistically significant $(P=0.025)$ (Supplementary Digital Content, Tables 2 and 3, http://links.lww.com/SCS/C682).

The patients were divided into three groups according to the day the MCC test was performed, and the relationship between these values and MCC was evaluated, and no statistically significant difference was found $(P=0.239)$ (Supplementary Digital Content, Tables 2-4, http://links.lww.com/SCS/C682).

When CT involvement type and on which day of the disease the test was performed and whether there is a correlation between the test and MCC was examined, no significant correlation was found between them ( $P$ values $P=0.225$ and $P=0.239$, respectively) (Supplementary Digital Content, Table 2, http://links.lww.com/ SCS/C682)

\section{DISCUSSION}

The coronavirus was first discovered by Tyrell and Bynoe in patients with the common cold in $1966 .{ }^{12}$ Coronaviruses cause colds with mild to moderate symptoms in the head and neck region. Although alpha coronaviruses typically cause asymptomatic or mildly symptomatic infections, beta coronaviruses (the group containing SARS-CoV-2) cause severe respiratory disease and death. In the case of SARS-CoV-2, the coronavirus-related disease has been named COVID-19. ${ }^{13}$

Anorexia, shortness of breath, mucus production, muscle, and joint pain have been reported in more than $25 \%$ of cases, with fever, fatigue, and dry cough being the most common symptoms at the onset of COVID-19 infection in hospitalized patients in China. ${ }^{1,13}$

Sore throat, rhinorrhea, headache, nausea, and diarrhea are less common, mostly in mild or moderate disease forms. ${ }^{1}$

Cough, shortness of breath, sore throat, rhinorrhea, nasal congestion, throat congestion, tonsil edema, enlarged cervical lymph nodes, or dizziness are symptoms that an otolaryngologist may encounter when examining COVID-19 patients. ${ }^{1,2}$

In the meta-analysis of Lovato et $\mathrm{al}^{2}{ }^{2}$ when evaluated in terms of otolaryngology symptoms, they reported that sore throat $(12.4 \%)$, nasal congestion $(3.7 \%)$, and rhinorrhea were the most common symptoms among patients with COVID-19.

By the literature, the most common symptoms in our patients were fever $(24.7 \%, \mathrm{n}=25)$, fatigue $(19.8 \%, \mathrm{n}=20)$, cough $(16.8 \%$, $\mathrm{n}=17)$, and dyspnea $(13.8 \%, \mathrm{n}=14)$. Loss of taste and smell $(9.9 \%, \mathrm{n}=10)$, nasal congestion $(6.9 \%, \mathrm{n}=7)$, runny nose $(4.9 \%, n=5)$, sore throat $(2.9 \%, n=3)$ are among the rare upper respiratory tract symptoms we observed in our patients.
Mucociliary clearance is an important defense mechanism in the protection of the human upper and lower respiratory tract against pathogens, foreign bodies, and toxins. For this reason, evaluating nasal MCC activity has been the goal of many researchers. The saccharin test is one of the methods used in MCC measurement. ${ }^{6,14}$ Our study used the saccharin clearance test to evaluate MCC because it is easy to apply, has a low incidence of side effects, and low cost.

Prolonged MCC duration indicates decreased mucociliary clearance. According to Anderson and Proctor, ${ }^{15}$ a nasal MCC longer than 30 minutes may indicate an abnormal disease that requires further investigation to diagnose pathologies related to mucus or clearance mechanisms. Stanley et $\mathrm{al}^{16}$ stated that prolonged MCC needs to be investigated to diagnose pathologies related to mucus or clearance mechanisms. Both acquired and genetic impairment of MCC disrupt respiratory mechanisms and cause chronic infections of the nose, paranasal sinuses, and lower respiratory tracts. ${ }^{14}$

It is known that viral infections cause a change in MCC by increasing mucus secretion, affecting normal ciliary movement, and causing airway epithelial damage. In the literature, studies are showing the change of MCC duration in viral diseases such as Human Immunodeficiency Virus (HIV) and Crimean-congo hemorrhagic fever (CCHF). ${ }^{14,17}$

Milgrim et $\mathrm{al}^{17}$ reported that the duration of nasal MCC in HIV patients was longer than in nonHIV controls, and the difference between them was statistically significant. The authors stated that the prolonged nasal MCC duration correlated with nasal obstruction.

Durmus et $\mathrm{al}^{14}$ found that nasal MCC duration was longer in patients with a diagnosis of laboratory-confirmed CCHF than healthy individuals and that the difference between them was statistically significant. They reported that clinicians should follow CCHF patients more closely for respiratory tract diseases, sinonasal and tympanum infections.

Based on this information in the literature, we evaluated the MCC duration in adult patients with COVID-19 and found no statistically significant difference between the groups $(P=0.760)$.

Smoking is one of the factors that negatively affect the MCC by reducing the normal cilia population, changing the mucus layer's viscoelasticity, and showing ciliotoxic effects. ${ }^{6,7,16}$

A study by Vastag et $\mathrm{al}^{18}$ on smoking patients reported that MCC was worse in smoking patients with chronic bronchitis.

In the study of Stanley et al, ${ }^{16}$ nasal MCC durations of smoking and nonsmoking patients were measured and compared with the saccharin test. They found that the mean nasal MCC duration of smoking patients was 20.8 minutes, whereas the mean nasal MCC duration of nonsmoking patients was 11.1 minutes, and concluded that smoking prolonged the nasal MCC duration.

In a study by Karaman et $\mathrm{al}^{6}{ }^{6}$ in which the relationship between septum deviation and smoking with nasal MCC was evaluated via the saccharin test, they reported that both factors prolong the nasal MCC duration.

In the study of Özler et $\mathrm{al}^{7}{ }^{7}$ evaluating the effect of passive and active smoking on nasal MCC duration, they reported that the mean nasal MCC duration was significantly longer in the passive and active smoking groups compared to the healthy group. They did not find a statistically significant difference in mean nasal MCC duration between the passive and active smoking groups. They reported that exposure to tobacco was associated with asthma, chronic rhinosinusitis, and lower respiratory tract infections, which may be associated with prolonged nasal MCC duration.

Our study found that smoking increases MCC, but we could not find a statistically significant difference between the groups.

The COVID-19 disease is an acute viral infection, and viral infections are known to impair mucociliary functions. This study is also important because it is the first study to detect changes in 
mucociliary clearance time in patients with COVID-19 in the adult population and compare them with healthy individuals.

One of the important limitations of our study is the relatively small number of patients. We think that studies with a larger sample size may contribute to further knowledge. In our study, we grouped the patients as smokers and nonsmokers. However, we did not evaluate the relationship between smoking exposure duration and MCC. However, as the exposure time to cigarettes increases, the MCC duration may be affected. We preferred to use the saccharin test in our study because it is cheap and easy to apply. The saccharin test is subjective and is evaluated according to the patient's responses. Using more objective testing to measure MCC duration could make our study more reliable. We applied the MCC test in COVID-19 patients who were followed in the service and had mild and moderate symptoms. Since the test we used requires patient adherence, we believe that a testing method that can be applied to patients with severe complaints and patients hospitalized in the intensive care unit may change our study results.

\section{CONCLUSIONS}

Coronavirus disease 2019 is a disease of increasing importance in many countries, including Turkey. It is a disease that reveals lower respiratory tract symptoms such as dyspnea and cough, as well as general symptoms such as fever and fatigue. Nasal congestion, runny nose, sore throat, loss of taste, and smell are rare upper respiratory tract symptoms in COVID-19. We think that the disease does not significantly change the MCC, which evaluates the upper respiratory tract activity, since it mostly affects the lower respiratory tract. We think that smoking contributes to the deterioration of mucociliary clearance in the upper respiratory tract, causing a further increase in lower respiratory tract symptoms and a worse disease course in COVID-19 patients.

\section{REFERENCES}

1. Krajewska J, Krajewski W, Zub K, et al. COVID-19 in otolaryngologist practice: a review of current knowledge. European Archives of OtoRhino-Laryngology

2. Lovato A, de Filippis C. Clinical Presentation of COVID-19: A Systematic Review Focusing on Upper Airway Symptoms. Ear, Nose \& Throat Journal 1-8a The Author(s) 2020

3. Fiorillo L, Cervino G, Matarese M, et al. COVID-19 surface persistence: a recent data summary and its importance for medical and dental Settings. Int J Environ Res Public Health 2020;17:3132

4. Cavallo L, Marcianò A, Cicciù M, et al. 3D Printing beyond Dentistry during COVID 19 epidemic: a technical note for producing connectors to breathing devices. Prosthesis 2020;2:46-52

5. Cervino G, Oteri G. Covid 19 pandemic and telephone triage before attending medical office: problem or opportunity? Medicina 2020;56:250

6. Karaman M, Tek A. Deleterious effect of smoking and nasal septal deviation on mucociliary clerance and improvement after septoplasty. Am J Rhinol Allergy 2009;23:2-7

7. Özler GS, Şimşek GÖ, Akbay E, et al. The effect of passive and active smoking on nasalmucociliary clerarance time. J Clin Anal Med 2016;7:149-151

8. Andersen I, Camner P, Jensen PL, et al. Nasal clearance in monozygotic twins. Am Rev Respir Dis 1974;110:301-305

9. Rutland J, Cole PJ. Nasal mucociliary clearance and ciliary beat frequency in cystic fibrosis compared with sinusitis and bronchiectasis. Thorax 1981;36:654-658

10. Duran M, Ülkü ÇH. Izole alt konka hipertrofisi olan hastalarda radyofrekans termal ablasyon tedavisinin nazal mukosilier klirens üzerine etkisi. Kulak Burun Bogaz Ihtis Derg 2014;24:185-189

11. Alho OP. Nasal airflow, mucociliary clearance, and sinus functioning during viral colds: effects of allergic rhinitis and susceptibility to recurrent sinusitis. Am J Rhinol 2004;18:349-355

12. Tyrrell DA, Bynoe ML. Cultivation of viruses from a high proportion of patients with colds. Lancet 1966;1:76-77
13. Sapideh Gilania, Rachel Roditib, Mohsen Naraghi COVID-19 and anosmia in Tehran, Iran. Medical Hypotheses 141(2020) 109757

14. Durmus K, Engin A, Karatas? TD, Gözel MG, Altuntas EE. Determination of Nasal Mucociliary Clearance Time and Nasal Symptom in Patients with Crimean-Congo Hemorrhagic Fever. J Med Virol

15. Andersen I, Proctor DF. Measurement of nasal mucociliary clearance. Eur J Respir Dis Suppl 1983;127:37-40

16. Stanley P, MacWilliam L, Greenstone M, et al. Efficacy of a saccharin test for screening to detect abnormal mucociliary clearance. $\mathrm{Br} J \mathrm{Dis}$ Chest 1984;78:62-65

17. Milgrim LM, Rubin JS, Small CB. Mucociliary clearance abnormalities in the HIV infected patient: aprecursor to acute sinusitis. Laryngoscope 1995:1202-1208

18. Vastag E, Matthys H, Zsamboki G, et al. Mucociliary clearance in smokers. Eur J Respir Dis 1986;68:107-113

\section{Jaffe Lichtenstein Type of Polyostotic Skeletal Fibrous Dysplasia With the Involvement of Cranial Bones}

\author{
Ozlem Akkemik, MD, ${ }^{*}$ Serkan Erkus, MD, ${ }^{\dagger}$ \\ Onder Kalenderer, $M D{ }^{\ddagger}$ and Nesrin Dündar, $M D^{\S}$
}

\begin{abstract}
Fibrous dysplasia (FD), a benign fibro-osseous bone tumor commonly involving the craniofacial region, presents a wide spectrum of radiographic patterns. When FD lesions are suspected, various imaging modalities and histopathologic analyses may be required to reach a firm diagnosis. Here, the authors report the case of a 10 -year-old male patient presenting with polyostotic type of FD with follow-up data for the next 9 years. The patient showed involvement of cranial bones and bilateral femurs, with skin pigmentation and no endocrine disease, consistent with the diagnosis of Jaffe Lichtenstein disease. The craniofacial radiographic features obtained with computed tomography and cone-beam computerized tomography are documented in this article.
\end{abstract}

Key Words: Bone tumor, dental, fibrous dysplasia

E ibrous dysplasia (FD) is an idiopathic, nonhereditary, sporadic, non-neoplastic tumor-like congenital process of the bone, which is replaced by fibro-osseous tissue. This leads to an expansion of the

From the *Department of Maxillofacial Radiology, Faculty of Dentistry, Yeni Yuzyil University, Istanbul; †Department of Orthopedics and Traumatology, Van Training and Research Hospital, Van; †Department Orthopedics and Traumatology, Tepecik Training and Research Hospital; and §Department of Maxillofacial Radiology, Faculty of Dentistry, Ege University, Izmir, Turkey.

Received November 1, 2020.

Accepted for publication February 20, 2021.

Address correspondence and reprint requests to Serkan Erkus, MD, Department of Orthopedics and Traumatology, Van Training and Research

Hospital, 65170, Van, Turkey; E-mail: s.erkus@ymail.com

The authors report no conflicts of interest.

Copyright $\odot 2021$ by Mutaz B. Habal, MD

ISSN: $1049-2275$

DOI: $10.1097 /$ SCS.0000000000007700 